\title{
Publication des études Women's Health Initiative Memory Study et Million Women Study
}

Swissmedic

1 Swissmedic. Publication des résultats intermédiaires de l'étude Women's Health Initiative. Bull Méd Suisses 2002;83(47):2548-50.

2 Writing Group for the Women's Health Initiative Investigators. Risks and benefits of estrogen plus progestin in healthy postmenopausal women. JAMA 2002; 288(3):321-33.

3 Shumaker et al. Estrogen plus progestin and the incidence of dementia and mild cognitive impairment in postmenopausal women: the Women's Health Initiative Memory Study: a randomized controlled trial. JAMA 2003;289(20):2651-62

4 Million Women Study Collaborators. Breast cancer and hormonereplacement therapy in the Million Women Study. Lancet 2002; 362:419-27.

Correspondance:

Swissmedic

Erlachstrasse 8

CH-3012 Berne 9

\section{Introduction}

En novembre 2002 déjà, Swissmedic publiait une prise de position [1] au sujet de l'analyse intermédiaire de l'étude Women's Health Initiative (WHI) [2], une étude contrôlée contre placebo, randomisée, qui examinait le bénéfice et le risque de la thérapie hormonale substitutive (THS) chez les femmes ménopausées. Le traitement consistait en estrogènes conjugués équins à la dose journalière de $0,625 \mathrm{mg}$ et acétate de medroxyprogestérone à la dose journalière de 2,5 mg. Les femmes hystérectomisées recevaient l'estrogène seul, les femmes non hystérectomisées recevaient l'association. Le bras de l'étude correspondant au traitement combiné fut arrêté prématurément, un risque plus élevé de carcinome mammaire invasif, de maladie coronarienne, d'attaque vasculaire cérébrale et de thromboembolie ayant été constaté en comparaison avec le placebo. L'analyse intérimaire n'ayant pas signalé de problèmes de sécurité pour la monothérapie, cette partie de l'étude est poursuivie.

Les résultats de la nouvelle étude Women's Health Initiative Memory Study (WHIMS) [3] ont été publiés dans le Journal of the American Medical Association (JAMA) le 28 mai 2003, et ceux de la Million Women Study [4] dans le Lancet du 9 août 2003. Ces études fournissent une information importante sur la sécurité de la THS. En tant qu'autorité de contrôle des médicaments, Swissmedic souhaite informer les milieux concernés de ces résultats.

\section{Résumé des résultats des études}

4532 femmes ont pris part à l'étude WHIMS, une étude partielle du projet WHI. Au début de l'étude, $46 \%$ des femmes étaient âgées de 65 à 69 ans, environ $36 \%$ de 70 à 74 ans et environ $18 \%$ étaient âgées de plus que 75 ans. Aucune des femmes ne souffrait de démence au moment du début de l'étude. Les critères primaires concernant les risques étaient la survenue d'une démence probable (probable dementia) ainsi que l'apparition d'une dysfonction cognitive modé- rée (mild cognitive impairment). Pendant la durée de l'étude de quatre ans en moyenne, 61 femmes ont développé une démence probable (40 dans le groupe de traitement hormonal et 21 dans le groupe placebo), ce qui donne une élévation relative du risque de 2,05 (intervalle de confiance $95 \%, 1,21-3,48)$. Ceci signifie en chiffres absolus 23 cas supplémentaires par an pour 10000 femmes traitées. La différence entre THS et placebo était déjà observable un an après le début de l'étude et augmentait au cours du temps. Contrairement à la démence probable, la dysfonction cognitive modérée était observée à la même fréquence dans les deux groupes de traitement. Il n'est pour l'instant pas clair si cette observation est aussi valable pour d'autres préparations de THS combinées. En Suisse, l'association fixe d'estrogènes équins et d'acétate de medroxyprogestérone mentionnée ci-dessus est autorisée uniquement dans le traitement des symptômes d'une carence estrogénique due à une ménopause naturelle ou artificielle chez des patientes non hystérectomisées et n'est ainsi dans la règle pas utilisée par des patientes de plus de 65 ans.

L'étude Million Women Study, une étude de cohorte non randomisée, a recruté 1084110 femmes britanniques de 1996 à 2001 dans le cadre du National Health Service Breast Screening Programme. En même temps que la convocation à la mammographie de dépistage, les participantes recevaient un questionnaire concernant les facteurs démographiques et l'utilisation d'une THS. L'âge moyen des femmes lors de l'entrée dans l'étude était de 55,9 ans. La moitié des femmes recevaient une THS avant ou lors de l'entrée dans l'étude, les autres femmes n'avaient jamais reçu de THS. Les critères primaires de sécurité étaient le diagnostic d'un cancer du sein et la mortalité suite à cette maladie. 9364 cas de carcinome mammaire invasif et 637 décès consécutifs à un cancer du sein ont été enregistrés après une durée moyenne d'observation de 2,6 et 4,1 ans, respectivement. Les femmes qui utilisaient une THS au moment de l'entrée dans l'étude montraient un risque significativement plus élevé que celui des femmes jamais traitées, aussi bien en terme de morbidité que de mortalité due au cancer du 
5 Collaborative Group on Hormonal Factors in Breast Cancer. Breast cancer and hormone replacement therapy: collaborative reanalysis of data from 51 epidemiological studies of 52,705 women with breast cancer and 108,411 women without breast cancer. Lancet 1997;350:1047-59. sein. Le risque relatif était de 1,66 (1,58-1,75, p $<0,0001)$ pour la morbidité et de 1,22 (1,00-1,48, $\mathrm{p}=0,05)$ pour la mortalité. Le risque de développer un cancer du sein était élevé lors de l'utilisation d'estrogènes seuls $(1,30[1,21-1,40])$, d'association estrogène-gestagène $(2,00[1,88-2,12]$ et de tibolone $(1,45[1,25-1,68])$, avec le risque le plus élevé sous thérapie combinée. Pour différents estrogènes et gestagènes, différents dosages ainsi que pour les thérapies continues ou séquentielles, les résultats étaient comparables. Le risque relatif était significativement plus élevé pour les formulations orales, transdermales et implantables $(1,32$ [1,21-1,45], 1,24 [1,11-1,39] respectivement $1,65[1,26-2,16])$. Pour toutes les modalités de la THS, le risque augmentait avec la durée d'utilisation.

Les résultats de la Million Women Study sont compatibles avec ceux de l'étude WHI et d'une métaanalyse déjà publiée précédemment [5], qui se basait sur 51 études épidémiologiques. Ces deux études plus anciennes décrivent également un risque plus élevé de cancer du sein sous THS. Il convient toutefois d'observer que la Million Women Study, contrairement à l'étude WHI, est une étude de cohorte non randomisée et qu'un biais éventuel ne peut pas être exclu avec certitude. Par contre, les points forts de cette étude sont sa taille et sa proximité à la réalité clinique.

\section{Conclusions et suite de la procédure}

Les textes d'information médicale des préparations de THS disponibles en Suisse contiennent déjà une mise en garde relative à l'augmentation du risque de carcinome mammaire sous THS et ont été retravaillés sur la base des résultats de l'étude WHI. Nous soulignons dans ce contexte que l'information médicale remaniée recommande aux médecins de prendre en compte les résultats de l'étude WHI ainsi que les risques précédemment connus avant de prescrire une THS. Pour les patientes à risque de carcinome mammaire, de thromboembolie ou d'accidents vasculaires ou cérébrovasculaires, d'autres thérapies devraient si possible être envisagées.

En collaboration avec des experts externes et les associations médicales concernées, Swissmedic évalue en ce moment de futures mesures supplémentaires destinées à limiter les risques, sur la base des résultats des études WHIMS et Million Women Study. En outre, Swissmedic requerra la prise de position des entreprises pharmaceutiques concernées. 